\title{
The stochastic effects of Ly $\alpha$ absorbers on the broadband luminosities of galaxies
}

\author{
Thorsten Tepper-García ${ }^{1} \dagger$, \\ and Uta Fritze-v. Alvensleben ${ }^{1}$ \\ ${ }^{1}$ Uni-Sternwarte Göttingen, Geismarlandstr. 11, 37083 Göttingen, Germany. \\ email: tepper,ufritze@astro.physik.uni-goettingen.de
}

\begin{abstract}
We investigate the variations in the broadband luminosities of low and intermediate redshift galaxies due to the stochastic nature of the neutral hydrogen distribution present in the form of Ly $\alpha$ absorbers in the intergalactic medium. This effect is caused by variations in the distribution and properties (redshift, column density, Doppler parameter) of the absorbers along different lines-of-sight out to a given redshift. Using a set of observationally constrained redshift-, column density-, and Doppler parameter distributions we perform Monte Carlo simulations for a large number of lines-of-sight towards galaxies at a given redshift $z_{e m}$ and calculate attenuated FUV/NUV magnitudes and corresponding 1-, 2-, and $3 \sigma$ variations. We predict significant variations in luminosity ranging from -0.22 to +0.48 magnitudes at the $1 \sigma$ level for galaxies at $z=1.5$ in $G A L E X$ data.
\end{abstract}

\section{Introduction}

The observed photometric properties of local and distant galaxies are important to constrain evolutionary synthesis models describing galaxy formation and evolution. But estimating colours and luminosities of galaxies from models and comparing them to the observed ones is a difficult task since, among other effects, the light of a galaxy is partially absorbed by the intervening neutral hydrogen (HI) present in the intergalactic medium (IGM) in form of discrete Ly $\alpha$ absorbers. The distribution of these Ly $\alpha$ absorbers along the line-of-sight (LOS) is stochastic in nature, i.e. the population of Ly $\alpha$ absorbers characterised by their redshift $z_{a b s}$, column density $N_{\mathrm{HI}}$, and Doppler parameter $b$ is unique for each LOS. Since the observation of individual galaxies implies necessarily the observation along different lines-of-sight, we expect that the stochastic nature of the Ly $\alpha$ absorbers distribution, especially of those at the highest column densities, should cause a significant scatter in the observed broadband colours, even for galaxies with similar intrinsic colours. Furthermore, we expect these stochastic effects to be strongest along the shortest LOSs and, hence, to be only observable in the UV as e.g. with GALEX.

In order to correctly account for the absorption of light caused by this intervening absorbing HI-systems, a detailed knowledge of their properties (i.e. $z_{a b s}, N_{\mathrm{HI}}, b$ ) is required. It has been found from statistical analyses of quasar absorption lines that when averaged over a large number of LOSs, the mean distribution and properties of Ly $\alpha$ absorbers are well described by a redshift- and column density-dependent power law of the form of Eq. 2.1 (see e.g. Kim et al. 1997, and references therein). Using different sets of parameters for this power law derived from observations, there have been various studies (see e.g. Møller \& Jakobsen 1990; Madau 1995; Bershady, Charlton \& Geoffroy 1999, hereafter BCG99), which, with different purposes and approaches, have made an effort to account for the mean attenuation of the light of distant galaxies due to intervening Ly $\alpha$

$\dagger$ Present address: Uni-Sternwarte Göttingen, Geismarlandstr. 11, 37083 Göttingen, Germany. 
absorbing systems and the dispersion around this mean. In this work, we make a further effort to calculate the stochastic effect of the Ly $\alpha$ absorbers on the photometric properties of galaxies. Applying some of the methods developed by Møller \& Jakobsen (1990) and BCG99, we recover the formalism of Madau (1995), i.e. we model the mean attenuation and any desired $\sigma$ level around this mean as a function of redshift. The advantage of our approach is that it is of general use in that it is completely independent of the galaxy input spectrum, and that it includes the corrections concerning the calculation of the $\sigma$ scatter pointed out by BCG99. As an application of our model, we estimate mean magnitudes and scatter at the 1-, 2-, and $3 \sigma$ level in the GALEX FUV/NUV broadband filters for galaxies with flat input spectra at $0.3<z_{e m}<1.5$.

\section{The model}

We present Monte Carlo simulations for the distribution of intervening HI-systems along random LOSs out to a given redshift $z_{e m}$ (see also Møller \& Jakobsen 1990; BCG99). As input we use differential distributions for the number density of clouds as a function of redshift and their column densities of the form

$$
\frac{\partial^{2} \mathcal{N}}{\partial N_{\mathrm{HI}} \partial z}=\mathcal{N}_{0} \cdot(1+z)^{\gamma} \cdot N_{\mathrm{HI}}^{-\beta}
$$

and a redshift-dependent Gaussian distribution for the Doppler parameters, all derived from observations (Kim et al. 1997; BCG99). We generate an ensemble of $4 \times 10^{3}$ LOSs out to a given $z_{e m}$, each of them containing a random absorber population drawn from the input distributions. For each absorber along each LOS we calculate the photoelectric and Lyman-series absorption assuming Voigt-profiles and a flat input spectrum with rest-frame resolution $\Delta \lambda=0.5 \AA$. We obtain a set of transmission values at each point of our wavelength baseline and get in this way an ensemble of transmission values in the range $[0,1]$ as a function of wavelength.

On this basis we define the Transmission Function $T\left(e^{-\tau} \mid \lambda ; z_{e m}\right)$ as the residual transmission along a random LOS out to $z_{e m}$ at a given wavelength $\lambda$ as a function of the absorption coefficient $e^{-\tau}$. It gives, up to a normalisation constant, the probability for the absorption coefficient $e^{-\tau}$ to take a certain value in the range $[0,1]$ at a given wavelength along a random LOS out to $z_{e m}$. Since there is no known analytic expression for $T\left(e^{-\tau} \mid \lambda ; z_{e m}\right)$, we determine it numerically from our simulations. It turns out that this distribution function is rather peculiar, and the calculation of relevant statistical moments for the absorption assuming Gaussian statistics may lead to incorrect estimates, as already noted by BCG99.

Let us define $\tau_{\mathrm{q}}$ as the $\mathrm{q}$ transmission quantile via the integral equation

$$
q=C_{0} \int_{0}^{\tau} \mathrm{q} T\left(1-\tau \mid \lambda ; z_{e m}\right) \mathrm{d} \tau,
$$

where $C_{0}$ is a normalisation constant and the mean value of $T\left(e^{-\tau} \mid \lambda ; z_{e m}\right)$ as $\tau_{50}$; and the $1-, 2-$, and $3 \sigma$ ranges around this value are $\tau_{50 \pm 34.13}, \tau_{50 \pm 47.72}$, and $\tau_{50 \pm 49.86}$ respectively. Using this definition we calculate mean attenuation curves (see Madau 1995) and the corresponding 1-, 2-, and $3 \sigma$ levels for any desired redshift, which can be applied to a given spectrum to estimate the amount of absorption due to the stochastic distribution of the Ly $\alpha$ absorbers and their properties (see Fig. 1). 


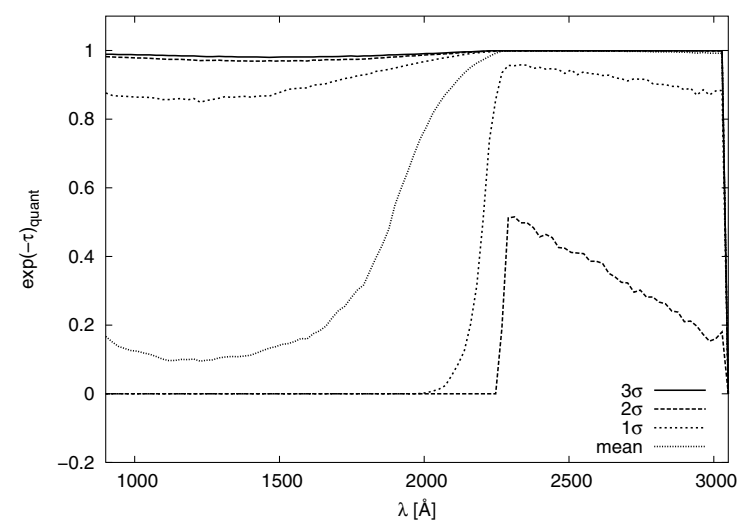

Figure 1. Attenuation curves for $z_{e m}=1.5$ : mean and 1-, 2-, and $3 \sigma$ variations (see Eq. 2.2). For clarity, the curve corresponding to 'mean $-3 \sigma$ ' is not shown.

\begin{tabular}{|c|c|c|c|c|c|}
\hline$z_{e m}$ & Passband & mean & $1 \sigma$ & $2 \sigma$ & $3 \sigma$ \\
\hline 0.3 & FUV & -18.82 & - & +0.13 & +1.16 \\
\hline 0.3 & NUV & -20.26 & $\overline{-}$ & +0.02 & +0.13 \\
\hline 0.5 & FUV & -18.69 & +0.02 & +0.28 & $=$ \\
\hline 0.5 & NUV & -20.25 & $=$ & +0.04 & +0.20 \\
\hline 1.0 & NUV & -20.25 & $\begin{array}{l}+0.19 \\
{ }_{-0.01}\end{array}$ & ${ }_{-0.01}^{+0.52}$ & ${ }_{-0.01}^{+1.27}$ \\
\hline 1.5 & NUV & -20.02 & $\begin{array}{l}+0.48 \\
{ }_{-0.22}\end{array}$ & $\begin{array}{l}+1.56 \\
{ }_{-0.24}\end{array}$ & $-\overline{-}$ \\
\hline
\end{tabular}

Table 1. Mean and 1-, 2-, and $3 \sigma$ variations in luminosity $\left(\mathrm{M}_{\mathrm{AB}}\right)$ in the GALEX FUV and NUV passbands for a flat input spectrum at four different redshifts $z_{e m} \in\{0.3,0.5,1.0,1.5\}$. In the cases where no value is given, either there is no significant variation or the flux inside the given filter has dropped to zero and the corresponding luminosity is not longer defined, being the case when the Lyman-break has already cross the filter or due to strong line absorption in the given filter.

\section{Preliminary results \& conclusions}

We have applied our method to calculate the effect of the Ly $\alpha$ absorbers on the FUV/NUV-broadband luminosities of a galaxy at redshifts $z \in\{0.3,0.5,1.0,1.5\}$. Our results are given in Table 1 . A careful inspection of these results shows that the contingent presence of Ly $\alpha$ clouds with the highest column densities can cause substantial variations in luminosity already at the $1 \sigma$ level, ranging from -0.22 to +0.48 magnitudes.

\section{Acknowledgements}

T. T. G. thanks the IAU, the Astronomische Gesellschaft (AG), and the BerlinerUngewitter Stiftung (BU) for partial travel support.

\section{References}

Bershady, M. A., Charlton, J. C., Geoffroy, J. M., 1999, ApJ, 518, 103

Kim, T.-S., Hu, E. M., Cowie, L. L., Songaila, A., 1997, AJ, 114, 1

Madau, P., 1995, ApJ, 441, 18

Møller, P., Jakobsen, P., 1990, A\&A, 228, 299 